\title{
Robust generation of entanglement between two cavities mediated by short interactions with an atom
}

\author{
Daniel E. Browne and Martin B. Plenio \\ Blackett Laboratory, Imperial College London, London SW7 2BW, United Kingdom
}

(Received 23 September 2002; published 31 January 2003)

\begin{abstract}
Current methods of preparing maximally entangled states in the laboratory need an extremely accurate control of interaction times, requiring sophisticated experimental techniques. Here, we show that such precise control is not necessary when one utilizes short or weak interactions followed by measurements. We present a scheme for the probabilistic generation of Bell states in a pair of cavities, after each has interacted briefly with an atom. The advantage of the scheme, as compared to present schemes, is its low sensitivity to the exact values of experimental parameters such as atomic velocity and coupling strength, in fact, for a large range of parameters, the fidelity of the Bell states generated remains close to unity.
\end{abstract}

DOI: 10.1103/PhysRevA.67.012325

PACS number(s): 03.67.-a, 42.50.-p, 03.65.Ud

\section{INTRODUCTION}

In recent years, entanglement, a fundamental feature of many-body quantum-mechanical systems, has come to be seen as a useful resource for many tasks in quantum information processing [1-3]. In particular, certain states have been identified, which can be directly used for many such tasks. Foremost among these are the so-called Bell states, also known in the literature as Einstein-Podolsky-Rosen (EPR) states, which exhibit the maximum entanglement that a two qubit system can possess. Such states are the fundamental ingredient of a variety of quantum information processing protocols (see Refs. [1-3] for details). Bell states are also of relevance to the study of the foundations of quantum mechanics. It was shown by Bell [4] that these states possess correlations that are incompatible with a local realistic theory (see Ref. [5] for a review). Thus, a loophole-free demonstration of such correlations would have profound implications for our understanding of the physical world. For these reasons, then, it is important to be able to generate Bell states in the laboratory, and many experimental proposals have been put forward and realized in both optical [6-8] and atomic systems $[9,10]$.

This paper contains a proposal for a scheme utilizing the techniques of cavity QED to generate Bell states between the modes of two spatially separated cavities. Rauschenbeutel and co-workers have generated Bell states between two modes in a single cavity using a Rydberg atom [11] coherently interacting with each mode in turn. This scheme could be adapted in a straightforward way to generate such states in spatially separated cavity modes. Cabrillo et al. have proposed a nondeterministic scheme to generate Bell entangled states between atoms conditionally, by driving them with a weak laser pulse, and subsequently detecting a photon spontaneously emitted by one of the atoms [12]. Protsenko and co-workers have then shown that this scheme can be adapted to implement quantum logic gates [13]. At the same time Plenio and co-workers have developed schemes to entangle atoms inside optical cavities and between atoms in different cavities via spontaneous decay [14-16]. Various other conditional schemes and deterministic schemes have been pro- posed, which employ the detection of photons to generate entanglement and perform quantum computation [17-19]. In schemes such as these, however, a very precise control of the experimental parameters is required. Such a precise control of experimental parameters is often difficult to achieve. Generally it is desirable to devise schemes in which the requirements of the experimental control are as small as possible. Such schemes would then be far more robust to errors and would generally lead to much improved fidelities of the generated states.

In this paper, we present a proposal for the robust experimental generation of a single-photon entangled Bell state $\left|\Psi^{+}\right\rangle=\sqrt{\frac{1}{2}}\left(|0\rangle_{A}|1\rangle_{B}+|1\rangle_{A}|0\rangle_{B}\right)$ in the modes of two spatially separated cavities, labeled $A$ and $B$. The cavity modes do not interact directly, but the generation of the entangled state is mediated by a resonant atom. This atom, prepared in its excited state, passes through the two cavities, prepared in their vacuum state, and interacts with each for the same short effective interaction time. The internal state of the atom is then measured. Depending on the outcome of this measurement, either a high-fidelity Bell state has been generated, or the cavities have returned to the vacuum state, and are ready for the process to be repeated with a fresh atom. The scheme has the advantage that provided the interaction times between the atom and both cavities are the same, which can be attained if a well-collimated atomic beam is utilized, the actual value of this interaction time can vary within a large range of values, without greatly affecting the fidelity of the Bell state produced. This would reduce the need for velocity selection of the atoms and for the sophisticated timing and feedback required to tune the interaction times in other experiments. Additionally, the interaction times, which this scheme would utilize, can be much shorter than in nonconditional schemes, which would reduce the effects of decoherence during the entanglement generation process.

In Sec. II we introduce the proposed scheme in detail. We begin by discussing the scheme in the limit where the effective duration of the atom-cavity interactions is very small, and the same in both cavities. We then discuss the fidelity of the state generated by a successful run of the scheme with finite interaction times and calculate the probability of a 


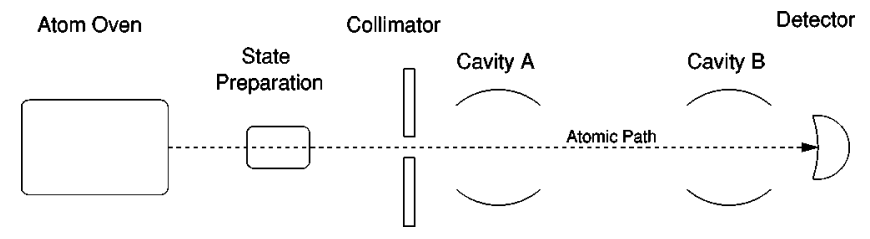

FIG. 1. A schematic diagram of the layout of the scheme.

single run of the scheme being successful. We find that, in a wide range of interaction times, the probability of success is suitably high, and the fidelity of the generated state is close to unity. In Sec. III we discuss practical aspects of implementing our scheme. In Sec. III A, we discuss specific physical systems with which the scheme could be implemented. In Secs. III A and III B, we consider two practical aspects of the scheme which would pose particular requirements to any implementation of the scheme. In Sec. III B we look at the effect on the scheme of inaccurate control of the path of the atom through the cavities due to poor collimation of the atomic beam. This would lead to unequal interaction times in the two cavities, potentially reducing the fidelity of the state generated. In Sec. III C, we discuss how employing detectors with less than perfect efficiency would affect the scheme.

\section{THE PROPOSED SCHEME}

In this section, we will describe the proposal that is the main focus of this paper-a scheme to generate the entangled Bell state $\left|\Psi^{+}\right\rangle$in two spatially separated microwave cavities. Two identical cavities, labeled $A$ and $B$, are positioned as in Fig. 1, such that their axes are parallel, and their centers are in alignment. The cavities must be prepared in their vacuum state. This can be achieved, for example, in microwave cavities by cooling to low temperatures. Twolevel atoms, whose transition between the ground state $|g\rangle$ and excited state $|e\rangle$ is resonant with modes of the cavities, are used to mediate the generation of the entangled state in the cavities. The atoms are prepared in their excited state $|e\rangle$. They are then passed, one at a time, through the center of both cavities, such that the effective interaction time with each cavity is much less than the period of a one-photon Rabi oscillation. Upon leaving the second cavity and before the next atom enters the cavities, the internal state of the atom leaving the cavities is measured. For brevity, in this paper, let us refer to the process of a single atom passing through both cavities and being measured as a "run" of the scheme. The outcome of the measurement is nondeterministic. If the excited state $|e\rangle$ is detected, then no entangled state has been generated in the cavities; we thus refer to a run that ends in such a measurement as a "null run." After a null run, the cavity is reset to its initial state, so a further run can be implemented immediately. If the ground state $|g\rangle$ of the atom is detected at the end of a run, a state is generated in the cavities, which, so long as certain conditions are fulfilled, as described below, is very close to the Bell state $\left|\Psi^{+}\right\rangle$. The process is complete at this point, and we shall thus label this a "successful run."

Before we describe the scheme in detail, let us summarize how the resonant interaction between an atom and a cavity is described. Consider a two-level atom with energy levels $|g\rangle$ and $|e\rangle$, separated by a transition energy resonant with a mode of the cavity. The atom is situated at position $r$ within the cavity, whose modes are described by field operators $a$ and $a^{\dagger}$. Since the atomic transition is resonant with the cavity mode, the internal atomic state interacts with this mode via the following Hamiltonian, in the interaction picture, where the rotating wave approximation has been employed:

$$
H=\hbar g_{0} u(\boldsymbol{r})\left(a^{\dagger}|g\rangle\langle e|+a| e\rangle\langle g|\right) .
$$

Here $g_{0}$ describes the strength of the coupling. When the atom is situated in the center of the cavity, the one-photon Rabi frequency is $g_{0} / \pi$. The spatial variation of the cavity field is contained in the mode function $u(\boldsymbol{r})$. If we consider an atom moving through the cavity on a classical path $\boldsymbol{r}(t)$, the mode function can then be written as a function of time $u(t)$. Since all the time dependence in $H$ is contained in $u(t)$, the unitary evolution of the system can be written as follows:

$$
U=\exp \left[-\frac{i\left(\int_{t_{0}}^{t} u\left(t^{\prime}\right) d t^{\prime}\right) \hat{H}}{\hbar}\right],
$$

where $\hat{H}=H / u(\boldsymbol{r})$ is the interaction Hamiltonian for an atom situated in the center of the cavity. In this paper, we will consider cases where the atom travels on a straight path through the cavity, and can thus introduce the total effective interaction time $\tau=\int_{-\infty}^{\infty} u\left(t^{\prime}\right) d t^{\prime}$ to characterize the interaction. The complete unitary evolution of the system due to the interaction $U_{\text {total }}$ is then

$$
U_{\text {total }}=\exp \left[-\frac{i \tau \hat{H}}{\hbar}\right] .
$$

In our scheme, the atom passes through and interacts with two cavities in turn, labeled $A$ and $B$. Let us label the interaction Hamiltonians for the interaction between the atom and each cavity $H_{A}$ and $H_{B}$, respectively. These take the same form as $H$ in Eq. (1) for each respective cavity. Analogously let us label the interaction Hamiltonians for the atom in the center of each cavity $\hat{H}_{A}$ and $\hat{H}_{B}$. The cavities are assumed to be identical and thus the coupling constant $g_{0}$ is the same for both. Let us consider how the state of the system will evolve if a resonant atom, initially in its excited state, passes through and interacts with the cavities, one after the other. If the cavities are initially in some pure state $\left|\psi_{\text {cav }}\right\rangle$, the initial state of the atom-cavities system is $|e\rangle\left|\psi_{\text {cav }}\right\rangle$. The atom passes through both cavities such that the effective interaction times are $\tau_{A}$ and $\tau_{B}$. After these interactions, the quantum state of the system has undergone a unitary evolution described by the operator $U_{A B}$,

$$
U_{A B}=e^{-i g_{0} \tau_{B} \hat{H}_{B}} e^{-i g_{0} \tau_{A} \hat{H}_{A}} .
$$

In the scheme we propose, the cavities are aligned as shown in Fig. 1 and the atom passes along the straight line through the centers of both cavities at constant velocity. This 
means that the effective interaction times between the atom and each cavity will be equal. It may, of course, be difficult to control the path of the atom with sufficient accuracy that the interaction times are exactly equal, and the effect of this is discussed in Sec. III B. For now, however, let us assume that $\tau_{A}$ and $\tau_{B}$ are equal, and label them both $\tau$. In the limit when the effective interaction times are very small, i.e., when $g_{0} \tau \ll 1, U_{A B}$ can be expanded to the first order in $g_{0} \tau$, and takes the following form:

$$
\begin{aligned}
U_{A B} & \approx 1-i \frac{H_{A} \tau}{\hbar}-i \frac{H_{B} \tau}{\hbar} \\
& =1-i g_{0} \tau\left[\left(a_{A}+a_{B}\right)|e\rangle\left\langle g\left|+\left(a_{A}^{\dagger}+a_{B}^{\dagger}\right)\right| e\right\rangle\langle g|\right] .
\end{aligned}
$$

The state of the system after the atom has left the second cavity is $|\psi\rangle=U_{A B}\left|\psi_{\text {init }}\right\rangle$ in this limit

$$
|\psi\rangle \approx|e\rangle\left|\psi_{\text {cav }}\right\rangle-i g_{0} \tau|g\rangle\left(a_{A}^{\dagger}+a_{B}^{\dagger}\right)\left|\psi_{\text {cav }}\right\rangle .
$$

When the state of the atom is now measured, the cavity modes are projected into one of two states, depending on the measurement outcome. If $|e\rangle$ is detected, the cavity returns to its initial state. This is important for a nondeterministic process, because it means that it can be repeated immediately from the same starting conditions. If the ground state $|g\rangle$ is detected, the cavity modes are now in the state, neglecting normalization, $\left(a_{A}^{\dagger}+a_{B}^{\dagger}\right)\left|\psi_{\text {cav }}\right\rangle$. Thus, if the cavities are initially in the vacuum state, the state generated in the cavities would be $\left(a_{A}^{\dagger}+a_{B}^{\dagger}\right)|0\rangle_{A}|0\rangle_{B}=|1\rangle_{A}|0\rangle_{B}+|0\rangle_{A}|1\rangle_{B}$, which, when normalized, is the Bell state $\left|\Psi^{+}\right\rangle$introduced above.

If, following a successful run, one were to repeat the scheme immediately and carry on until $n$ atoms had been detected in the ground state, in the limit that $g_{0} \tau$ is small, the state generated would have the form $\left(a_{A}^{\dagger}+a_{B}^{\dagger}\right)^{n}\left|\psi_{\text {cav }}\right\rangle$, or, if the cavities are initially in their vacuum state, $\left(a_{A}^{\dagger}\right.$ $\left.+a_{B}^{\dagger}\right)^{n}|0\rangle_{A}|0\rangle_{B}$. This is equivalent to the state produced when an $n$-photon Fock state and a vacuum state are incident together on a 50:50 beam splitter. However, numerical results have suggested that the fidelity of states produced via this method would decrease swiftly with increasing $n$. This is due to two reasons: first, the short interaction time approximation becomes worse when higher photon numbers are present in the cavities since the time scale of the interactions is faster (the Rabi frequency scales with $\sqrt{n+1}$ ); second, when more than one photon is in the cavities, a null run, the measurement of the atom leaving the cavities to be in its excited state, does not reset the state of the cavities to the state before the run, so, as more repetitions are made, the fidelity of the final state gets worse. For these reasons, this does not appear to be a good scheme for the generation of such states.

However, as we will show below, the generation of single-photon Bell states is not affected by these problems. First, we find that the fidelity of the generated state remains close to unity for values of $g_{0} \tau$ much greater than the above approximation would be valid. Second, in this case, null runs reset the state of the cavities to the vacuum state exactly, so the fidelity of the state generated is unaffected by the number

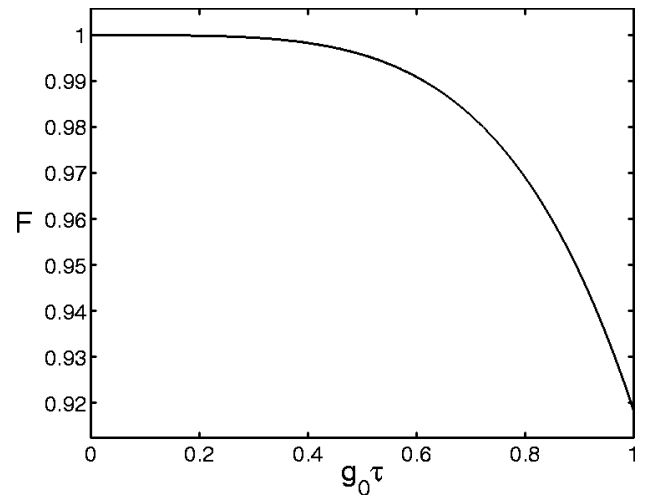

FIG. 2. The fidelity of the Bell state generated in the cavity modes after a successful run, plotted against $g_{0} \tau$ with values from 0 to 1 . Note that the values of $g_{0} \tau$ corresponding to a full Rabi oscillation in each cavity are $g_{0} \tau=\pi$.

of runs required. In the limit that $g_{0} \tau$ tends to zero, the probability of the detector measuring a ground state is $\approx 2\left(g_{0} \tau\right)^{2}$, which, in this limit, is vanishingly small. In a practical scheme, one will need to work in a parameter range where the probability of success is high enough that few repetitions are required to achieve a successful run. This is the case for higher values of $g_{0} \tau$, where the above approximation would no longer be valid. Fortunately, starting with the simple pure state $|e\rangle|0\rangle_{A}|0\rangle_{B}$, it is straightforward to solve the Schrödinger equation and calculate exactly the state of the system after the interactions have taken place. This state $|\psi\rangle$, under the assumption that the interaction times $\tau$ are exactly equal, is

$$
\begin{aligned}
|\psi\rangle= & \cos ^{2}\left(g_{0} \tau\right)|e\rangle|0\rangle_{A}|0\rangle_{B} \\
& -i \cos \left(g_{0} \tau\right) \sin \left(g_{0} \tau\right)|g\rangle|0\rangle_{A}|1\rangle_{B} \\
& -i \sin \left(g_{0} \tau\right)|g\rangle|1\rangle_{A}|0\rangle_{B} .
\end{aligned}
$$

Let us consider a measurement of atom's state. If the excited state is detected, the state of the cavities is projected back to the vacuum state, independent of $\tau$, as in the approximate case. This resets the cavities to their initial state, so the process can be immediately repeated with a fresh atom.

If the ground state is detected, the following entangled state is generated in the cavity:

$$
\left|\psi_{\text {cav }}\right\rangle=\cos \left(g_{0} \tau\right)|0\rangle_{A}|1\rangle_{B}+|1\rangle_{A}|0\rangle_{B},
$$

where normalization has been omitted. As $g_{0} \tau$ approaches zero, this tends to the desired state $\left|\Psi^{+}\right\rangle$. One can quantify how close this state generated is to $\left|\Psi^{+}\right\rangle$in terms of the fidelity $F=\left|\left\langle\Psi^{+} \mid \psi_{\text {cav }}\right\rangle\right|^{2}$ :

$$
F=\frac{1}{2}+\frac{\cos \left(g_{0} \tau\right)}{\cos ^{2}\left(g_{0} \tau\right)+1}=1-\frac{\left(g_{0} \tau\right)^{4}}{16}+O\left(\left[g_{0} \tau\right]^{6}\right) .
$$

This is plotted in Fig. 2. We see that the fidelity remains very close to unity for a surprisingly large range of $g_{0} \tau$. For example, the fidelity remains above $(1-4) \times 10^{-3}$ for $g_{0} \tau$ 


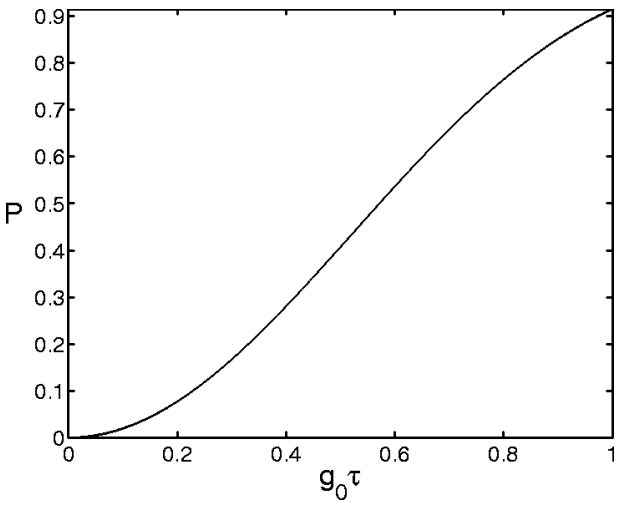

FIG. 3. The probability that a single run will lead to the detection of the atom in its ground state, and thus the successful generation of a Bell state, plotted for values of $g_{0} \tau$ from 0 to 1 .

$=0.5$. Additionally, the function is extremely flat in the wide range of values between $g_{0} \tau=0$ and $g_{0} \tau=0.5$. Therefore, the scheme would be insensitive to variations in the interaction times within this range.

For the scheme to be useful, the probability of a single run leading to the successful generation of an entangled state, $P_{\text {success }}$, needs to be high enough that prohibitively many repetitions are not required:

$$
P_{\text {success }}=1-\cos ^{4}\left(g_{0} \tau\right)=2\left(g_{0} \tau\right)^{2}+O\left(\left[g_{0} \tau\right]^{4}\right) .
$$

The success probability is plotted in Fig. 3. For $g_{0} \tau=0.5$, this probability is $\approx 0.4$, so a successful run would probably be achieved in two or three repetitions. The optimal parameter range for the scheme depends upon the fidelity of the state required. The higher the value of $g_{0} \tau$ chosen, the higher the success probability will be, but the lower the fidelity of the state generated. If, for example, fidelities of 0.95 were acceptable, and a minimum success probability of 0.5 were desired, the scheme could operate between $\approx g_{0} \tau=0.6$ and $g_{0} \tau=0.9$. The exact value of $g_{0} \tau$, however, can lie anywhere in this range, so no fine tuning of the interaction time is required. Note that even if extremely high fidelities such as 0.999 are required, the necessary parameters (up to $g_{0} \tau$ $=0.35$ ) still allow a success probability of up to 0.22 , meaning that a successful run would probably be reached after four or five repetitions.

\section{PRACTICAL CONSIDERATIONS}

\section{A. Implementation}

The above description is quite general and no particular type of atom or cavity has been specified. A number of aspects must be considered in the choice of a physical system to implement the scheme. First, it would be desirable that the entangled states, once generated, would be as long lived as possible. This favors microwave cavities over optical cavities, since the lifetime of a photon in an optical cavity is currently at the very most $1 \mu \mathrm{s}$ [20], whereas microwave cavities with a photon lifetime of $1 \mathrm{~m}$ have been made [21]. It takes an atom traveling at $500 \mathrm{~ms}^{-1}$ around $20 \mu \mathrm{s}$ to traverse a typical microwave cavity, which is much smaller than this lifetime; so there would be sufficient time for further atoms to probe and interact with the cavity mode before the entangled state has dissipated. If a microwave cavity is used, an atom with a microwave transition is then needed. Microwave transitions occur in atomic fine and hyperfine structure; however, dipole transitions between these states are forbidden, and their interaction with the cavity mode would be much too weak to implement this scheme-the interaction times that would be required would be much greater than the photon lifetime of the cavity. Rydberg atoms, on the other hand, although more difficult to prepare, have large dipole moments and thus would interact strongly with cavity modes.

Experiments have been carried out, for example by Haroche and co-workers [21], which have parameters close to that required in this scheme. Recall that our scheme requires that the product of parameters $g_{0}$ and $\tau$ is at the minimum 0.2 and maximally $0.5-0.8$, depending on the fidelity of Bell state one wants to generate. In the experiments in Ref. [21], Rydberg atoms and microwave cavity modes interact resonantly with a Rabi frequency of $47 \mathrm{kHz}$. This means that $g_{0}=47000 \pi \mathrm{s}^{-1}=1.48 \times 10^{5} \mathrm{~s}^{-1}$. Atoms in an atomic beam from an oven source travel at speeds of the order of hundreds of meters per second. In the experiment by Haroche and co-workers, atoms with a speed of $500 \mathrm{~ms}^{-1}$ are selected. This means that the effective interaction time is such that a single Rabi oscillation is performed, i.e., $g_{0} \tau$ $=\pi$. This is a factor of $4-8$ lower than the parameter range for our scheme. It would be difficult to lower $\tau$ by using faster atoms, since the velocity of the atoms scales with the square root of the atom oven temperature, so a lower $g_{0}$ would be required. This can be obtained be using a larger cavity, and since $g_{0}$ scales with $1 / \sqrt{V}=1 / L^{3 / 2}$, a cavity of mirror separation three or four times as great as in Ref. [21] experiments would lead to an effective interaction time in the required range. Thus Rydberg atoms and microwave cavities can be employed to implement the scheme.

The disadvantage in using Rydberg atoms is that, at present, the efficiency of state detection schemes is low. In Ref. [21], for example, they report a detection efficiency of $40 \%$. We will discuss the implications this has for the scheme in Sec. III C. First, however, we consider the effect on the fidelity of the entangled states produced in the scheme if the path of the atom through the cavities is not well controlled and deviates from the line through the centers of the cavities.

\section{B. The atomic path}

If the effective interaction times with both cavities are not the same, this can reduce the fidelity of the entangled state produced by a successful run of the scheme. In our discussion above, we assumed that the two interaction times were exactly equal. In practice, however, it could be difficult to control the path of the atom so precisely. Let us consider first the effect that differing interaction times would have in the fidelity of the Bell state generated by a successful run of the scheme. Let the effective interaction between the atom and 


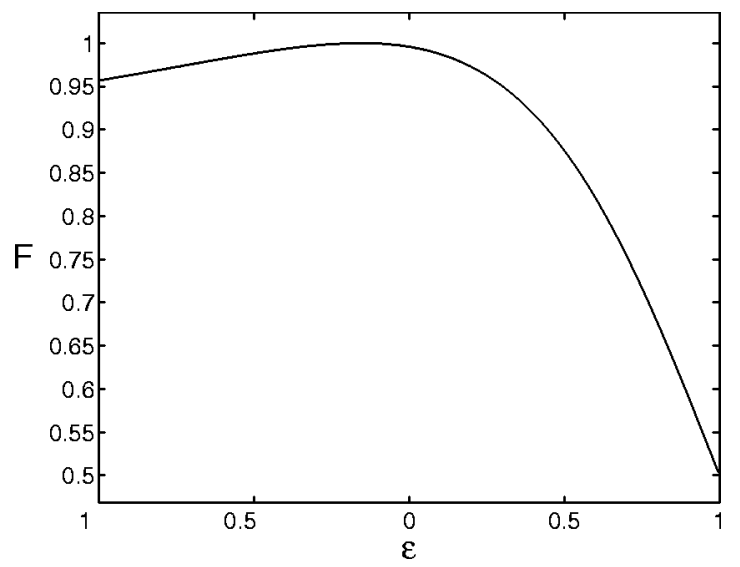

FIG. 4. The fidelity of the Bell state generated in the cavity, if the effective interaction times are $\tau$ and $\tau(1-\epsilon)$ for the interactions with cavities $A$ and $B$, respectively, is plotted here as a function of $\epsilon$ for $g_{0} \tau=0.5$.

cavity $A$ and the atom and cavity $B$ be $\tau$ and $\tau(1-\epsilon)$. We can rewrite equation (9), to take the differing interaction times into account, and find the following expression for the fidelity of the entangled state generated by a successful run of the scheme:

$$
F=\frac{1}{2}+\frac{\cos \left(g_{0} \tau\right) \sin \left(g_{0} \tau\right) \sin \left[g_{0} \tau(1-\epsilon)\right]}{\cos ^{2}\left(g_{0} \tau\right) \sin ^{2}\left[g_{0} \tau(1-\epsilon)\right]+\sin ^{2}\left(g_{0} \tau\right)}
$$

The fidelity is plotted for $g_{0} \tau=0.5$ as a function of $\epsilon$ in Fig. 4. The asymmetry of the plot is partly an artifact of the choice of parametrization, but, if we take this into account, by plotting $F$ again $\ln (1-\epsilon)$, as in Fig. 5, we see that the asymmetry remains. This is due to the asymmetry in the scheme itself regarding the interactions with the two cavities. When the atom enters the first cavity, it is always in the product state $|e\rangle$; whereas, when entering the second, it is always entangled with the first cavity. This makes the interaction with cavity $B$ in some sense, slightly weaker, and is the reason that the maximal value of $F$ occurs when $\epsilon$ has a

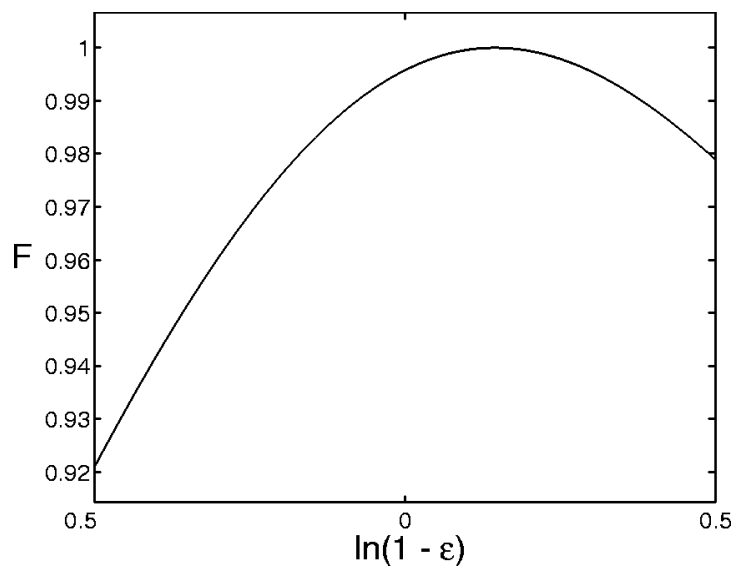

FIG. 5. The fidelity of the Bell state generated in the cavity plotted again as a function of $\epsilon$ for $g_{0} \tau=0.5$. In this case, plotted against $\ln (1-\epsilon)$.

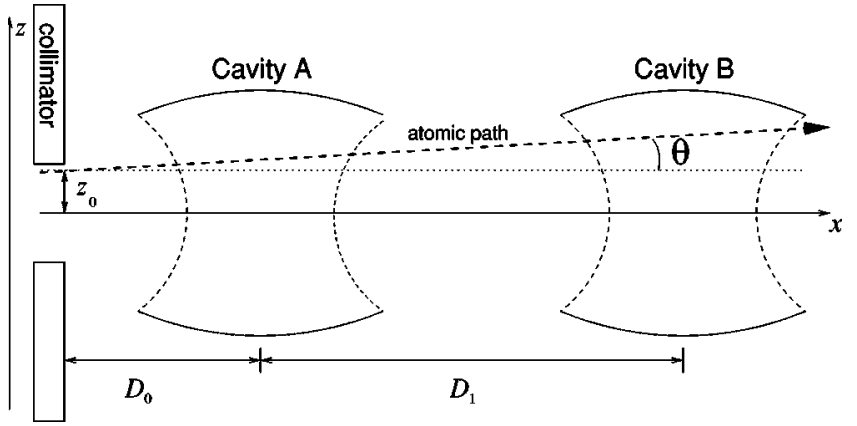

FIG. 6. A general straight-line path through the cavities can be defined in terms of the parameters $y_{0}, z_{0}, \phi$, and $\theta$, where distances are measured in meters and angles in radians. This figure illustrates $z_{0}$ and $\theta$. The other two parameters, $y_{0}$ and $\phi$, are equivalently defined in the perpendicular $x-y$ plane. The distance between the exit of the collimator and the center of cavity $A$ is $D_{0}$. The distance between the centers of the cavities is $D_{1}$.

small negative value. The slightly longer interaction time compensates for the interaction being slightly weaker.

Since it is reasonably easy to position the cavities in the desired place to very high precision, let us assume that they are perfectly aligned in the layout illustrated in Fig. 1. The factor that would be harder to control would be the atom's path through the apparatus, since it would be traveling on a ballistic path after being ejected from a heat oven, and although such atomic beams can be highly collimated, there will generally be a small amount of spread in the transverse direction, meaning that the atom's path will diverge slightly from the central path.

To calculate how much this would affect the interaction times we consider the cavity geometry. The spherical mirrors commonly used in cavity QED experiments support Gaussian cavity modes, which have the following mode function:

$$
u(x, y, z)=\exp \left[-\frac{x^{2}+y^{2}}{w_{0}^{2}}\right] \cos \frac{2 \pi z}{\lambda}
$$

where $w_{0}$ is the mode waist. The $z$ axis lies along the line connecting the centers of the two mirrors and the origin is in the center of the cavity. The waist of a Gaussian mode, $w_{0}$, is a function of the cavity geometry and the field wavelength,

$$
w_{0}=\left[\frac{\lambda \sqrt{L}}{2 \pi} \sqrt{R-L}\right]^{1 / 2} \text {, }
$$

where $L$ is the separation between the mirrors and $R$ is the radius of curvature of the mirrors. If the atom travels along the central axis of the cavity with constant speed $v$, the effective interaction time is $\sqrt{\pi} w_{0} / v$. Let us consider a general straight path through the system, which can be defined in terms of four parameters, $y_{0}$ and $z_{0}$, the initial displacements from the central line in the $y$ and $z$ directions, and $\phi$ and $\theta$ the angles between the atomic path and the central line in the $y$ and $z$ directions, illustrated for $z_{0}$ and $\theta$ in Fig. 6. We can calculate the effective interaction times between the atom and cavity as it travels along this path with speed $v$ and find 
that the interaction time with each cavity, in terms of displacements $\delta_{y}$ and $\delta_{z}$, which differ for each cavity, is

$$
\begin{aligned}
\tau_{\text {eff }}= & \frac{\sqrt{\pi} \omega_{0}}{v \cos \theta} \exp \left[-\frac{\delta_{y}^{2}}{\omega_{0}^{2}}\left(1-\frac{\sin ^{2} \theta}{\cos ^{2} \phi}\right)\right] \exp \left[-\frac{k^{2} \omega_{0}^{2} \tan ^{2} \theta}{4}\right] \\
& \times \cos \left[k \delta_{z}-k \delta_{y}\left(\frac{\sin \theta \sin \phi}{\cos ^{2} \theta}\right)\right] .
\end{aligned}
$$

For the interaction with cavity $A, \delta_{y}=y_{0}+\cos (\phi) D_{0}$ and $\delta_{z}$ $=z_{0}+\cos (\theta) D_{0}$; and for the interaction with cavity $B, \delta_{y}$ $=y_{0}+\cos (\phi)\left(D_{0}+D_{1}\right)$ and $\delta_{z}=z_{0}+\cos (\theta)\left(D_{0}+D_{1}\right)$. We can use these expressions to calculate $\epsilon$ in terms of these parameters, written here to the second order in $y_{0}, z_{0}, \phi$, and $\theta$ and their products,

$$
\begin{aligned}
\epsilon \approx & \frac{1}{w_{0}^{2}}\left[\left(D_{1} \phi\right)^{2}+\left(D_{1} \phi\right)\left(2 D_{0} \phi\right)+\left(2 y_{0}\right)\left(D_{1} \phi\right)\right] \\
& +\frac{2 \pi^{2}}{\lambda^{2}}\left[\left(D_{1} \theta\right)^{2}+\left(D_{1} \theta\right)\left(2 D_{0} \theta\right)+\left(2 z_{0}\right)\left(D_{1} \theta\right)\right] .
\end{aligned}
$$

Let us discuss the constraints this would have on the collimation of a typical experiment. In the cavity QED experiments of Haroche and co-workers [21,22], Rydberg atoms interact resonantly with microwave cavities. In a typical experiment, a cavity mode with $w_{0}=5.97 \mathrm{~mm}$ and $\lambda$ $=5.87 \mathrm{~mm}$ is employed. For $\epsilon$ to be small, the quantities in parentheses in Eq. (15) must be much smaller than $w_{0}$ and $\lambda / \sqrt{2} \pi=1.32 \mathrm{~mm}$. This means that the atomic beam must be collimated so that the effective beam radius is much smaller than this distance. In Ref. [22] an effective beam radius of $0.25 \mathrm{~mm}$ is reported. If we assume from this that, in the worst case, this would mean that $y_{0}, z_{0} \approx 0.25 \mathrm{~mm}$, and $D_{1} \phi, D_{1} \theta \approx 0.25 \mathrm{~mm}$, we can estimate that $\epsilon$ would be less than 0.2. If $g_{0} \tau=0.8$ and $\epsilon=0.2$, this would correspond to a reduction of the fidelity of the entangled state produced by a successful run from 0.96 if both interaction times are exactly 0.93 . Thus, with the beam collimation currently available in laboratory, Bell states with a fidelity high enough, for example, to exhibit significant violations of the Bell inequalities [5] could be generated.

Equation (15) also provides another reason why optical cavities would be unsuitable for the scheme. The typical waist of an optical cavity tends to be much smaller than that of a microwave cavity, so the demands on the atomic beam collimation required if optical cavities were used would be extremely high.

\section{Detector efficiency}

Our analysis in the preceding section assumes that the atomic state detector has perfect efficiency. In practice, the detection efficiency will be less than unity. Indeed, as mentioned above, current state detection methods for Rydberg atoms have an efficiency of just 40\% [21]. The detection process ionizes the atom, destroying the state, so increased efficiency cannot be obtained by placing detectors in series. A single detection failure will disrupt the scheme, since it will cause a mixed state to be created in the cavities. The scheme must then be halted, and one would then have to wait until this state dissipates from the cavities, and the cavities return to the vacuum state. Otherwise, if further atoms are sent through the cavities immediately, they will interact with the mixed state, and any "successful" run will generate a mixed state with much reduced fidelity. Rather than halting the flow of atoms through the cavities, their interaction with the cavities can be prevented for the cavity dissipation time by the application of an electric field to the system, to create a Stark shift in the atoms such that they are no longer resonant with the cavities. Therefore, one would like a detection efficiency high enough that the probability of a detection failure, during the typical number of runs needed before a successful ground-state measurement is made, is low. The mean number of runs to generate the entangled state in the cavity, if the detector were ideal, is simply the inverse of the success probability. The probability that the detector works every time during the process is therefore simply $D^{1 / P_{\text {success }} \text {, }}$ which for all values of $P_{\text {success }} \leqslant D$. Therefore, for a reliable scheme, a high detection efficiency would be desirable.

For example, let us consider an implementation of this scheme with Rydberg atoms traveling through the cavities such that $g_{0} \tau=0.5$. The success probability $P_{\text {success }}$ is $40.7 \%$ and with current detectors with efficiency $40 \%, P_{\operatorname{det}}$ would be around $10 \%$. This would mean, typically one would have to repeat the whole process, including preparation of the cavities, ten times before it could reach its successful conclusion. However, in light of the fact that the cavity dissipation time is of the order of milliseconds, and the time taken for each run much less than this, even in this case, the time needed to repeat the scheme enough times to generate the Bell state would be a fraction of $1 \mathrm{~s}$.

\section{CONCLUSION}

We have proposed a scheme for the generation of highfidelity Bell states between two spatially separated cavity modes. The scheme is nondeterministic, but we have shown that within the range of parameters $g_{0} \tau$ between $\approx 0.3$ and 0.9 , fidelities higher than 0.95 are obtained, with success probabilities for a single run greater than $1 / 5$ for this entire range.

The most appropriate physical system to implement this scheme is a combination of microwave cavities and Rydberg atoms. The low detection efficiency for Rydberg states would increase the number of times that the scheme would need to be repeated before a successful run, and require extra time after each detection failure to allow the mixed state produced in the cavities to dissipate. Nevertheless, the scheme would still be successful within a reasonable number of repetitions. The scheme requires that the atomic beam used is highly collimated, otherwise the fidelity of the states produced may be degraded, but the collimation, which has already been achieved in current experiments, is high enough that this effect would be small.

The principle of using brief interactions and measurement 
to generate entanglement can be adapted to many other physical systems, and may be especially useful in systems where interaction times are hard to control.

Note added: After this work was completed, we became aware of Ref. [23], in which the author presents a different scheme for the generation of Bell states in two cavities via the passage of a single atom through the cavities.

\section{ACKNOWLEDGMENTS}

This work was supported by EPSRC, the EQUIP project of the European Union, European Science Foundation program on "Quantum Information Theory and Quantum Computing," Hewlett-Packard Ltd. and U.S. Army Grant No. DAAD19-02-1-0161.
[1] M.A. Nielsen and I.L. Chuang, Quantum Computation and Quantum Information (Cambridge University Press, Cambridge, 2000).

[2] G. Alber, T. Beth, M. Horodecki, P. Horodecki, R. Horodecki, M. Rötteler, H. Weinfurter, R. Werner, and A. Zeilinger, Quantum Information, Springer Tracts in Modern Physics Vol. 173 (Springer, Berlin, 2001), Chap. 2.

[3] M. Plenio and V. Vedral, Contemp. Phys. 39, 431 (1998).

[4] J.S. Bell, Physics (Long Island City, N.Y.) 1, 195 (1964).

[5] L. Hardy, Contemp. Phys. 39, 419 (1998).

[6] C.S. Wu and I. Shaknov, Phys. Rev. 77, 136 (1950).

[7] P.G. Kwiat, K. Mattle, H. Weinfurter, A. Zeilinger, A.V. Sergienko, and Y. Shih, Phys. Rev. Lett. 75, 4337 (1995).

[8] A. Lamas-Linares, J.C. Howell, and D. Bouwmeester, Nature (London) 412, 887 (2001).

[9] M.A. Rowe, D. Kielpinski, V. Meyer, C.A. Sackett, W.M. Itano, C. Monroe, and D.J. Wineland, Nature (London) 409, 791 (2001).

[10] E.S. Fry, T. Walther, and S. Li, Phys. Rev. A 52, 4381 (1995).

[11] A. Rauschenbeutel, P. Bertet, S. Osnaghi, G. Nogues, M. Brune, J.M. Raimond, and S. Haroche, Phys. Rev. A 64, 050301 (2001).
[12] C. Cabrillo, J.I. Cirac, P. Garcia-Fernandez, and P. Zoller, Phys. Rev. A 59, 1025 (1999).

[13] I.E. Protsenko, G. Reymond, N. Schlosser, and P. Grangier, Phys. Rev. A 66, 062306 (2002).

[14] M.B. Plenio, S.F. Huelga, A. Beige, and P.L. Knight, Phys. Rev. A 59, 2468 (1999).

[15] S. Bose, P.L. Knight, M.B. Plenio, and V. Vedral, Phys. Rev. Lett. 83, 5158 (1999).

[16] E. Jané, M.B. Plenio, and D. Jonathan, Phys. Rev. A 65, 050302(R) (2002).

[17] E. Knill, R. Laflamme, and G.J. Milburn, Nature (London) 409, 46 (2001).

[18] P. Kok, H. Lee, and J.P. Dowling, Phys. Rev. A 65, 052104 (2002).

[19] J. Fiurasek, Phys. Rev. A 65, 053818 (2002).

[20] W. Lange (private communication).

[21] J.M. Raimond, M. Brune, and S. Haroche, Rev. Mod. Phys. 73, 565 (2001).

[22] M. Brune, F. Schmidt-Kaler, A. Maali, J. Dreyer, E. Hagley, J.M. Raimond, and S. Haroche, Phys. Rev. Lett. 76, 1800 (1996).

[23] A. Messina, Eur. Phys. J. D 18, 379 (2002). 